\title{
Curves with rational chord-length parametrization
}

\author{
J. Sánchez-Reyes ，L. Fernández-Jambrina \\ Instituto de Matemática Aplicada a la Ciencia e Ingenieria, ETS Ingenieros Industriales, Universidad de Castilla-La Mancha, \\ Campus Universitario, 13071-Ciudad Real, Spain \\ ETSI Navales, Universidad Politécnica de Madrid, Arco de la Victoria s/n, 28040-Madrid, Spain
}

\begin{abstract}
It has been recently proved that rational quadratic circles in standard Bézier form are parameterized by chord-length. If we consider that standard circles coincide with the isoparametric curves in a system of bipolar coordinates, this property comes as a straightforward consequence. General curves with chord-length parametrization are simply the analogue in bipolar coordinates of nonparametric curves. This interpretation furnishes a compact explicit expression for all planar curves with rational chord-length parametrization. In addition to straight lines and circles in standard form, they include remarkable curves, such as the equilateral hyperbola, Lemniscate of Bernoulli and Limaçon of Pascal. The extension to 3D rational curves is also tackled.
\end{abstract}

Keywords: Bézier circle; Bipolar coordinates; Chord-length parametrization; Equilateral hyperbola; Lemniscate of Bernoulli

\section{Chord-length parametrization}

Given a parametric curve $\mathbf{p}(t)$ over a certain domain $t \in[a, b]$, its chord-length at a given point $\mathbf{p}(t)$ is defined as (Farin, 2001, 2006):

$$
\operatorname{chord}(t):=\frac{|\mathbf{p}(t)-\mathbf{A}|}{|\mathbf{p}(t)-\mathbf{A}|+|\mathbf{p}(t)-\mathbf{B}|}, \quad \mathbf{A}=\mathbf{p}(a), \mathbf{B}=\mathbf{p}(b)
$$

where bars denote the modulus of a vector. The curve is said to be chord-length parameterized if chord $(t)=t$. Chordlength parametrization is clearly invariant with respect to linear transformations of the domain. Therefore, we may always reparameterize the curve as $\mathbf{p}(u)$ on a unit domain $u \in[0,1]$, so that $\mathbf{A}=\mathbf{p}(0), \mathbf{B}=\mathbf{p}(1)$.

Chord-length parametrization admits an intuitive physical interpretation (Fig. 1). Suppose you have a rubber band of unit length, on which we draw a unit scale representing the unit domain. Then we attach the rubber band to two fixed points $\mathbf{A}, \mathbf{B}$ and trace a curve by holding the band tout, pressing on it using a pencil without friction. As the band is submitted to a common tension all along its length, it stretches linearly, and hence condition (1) is satisfied. In fact, we could draw any curve using this method, but in general the curve would not be parametrized by a rational function of the chord $u$. The question to solve is precisely finding the curves for which the resulting parametrization is rational 

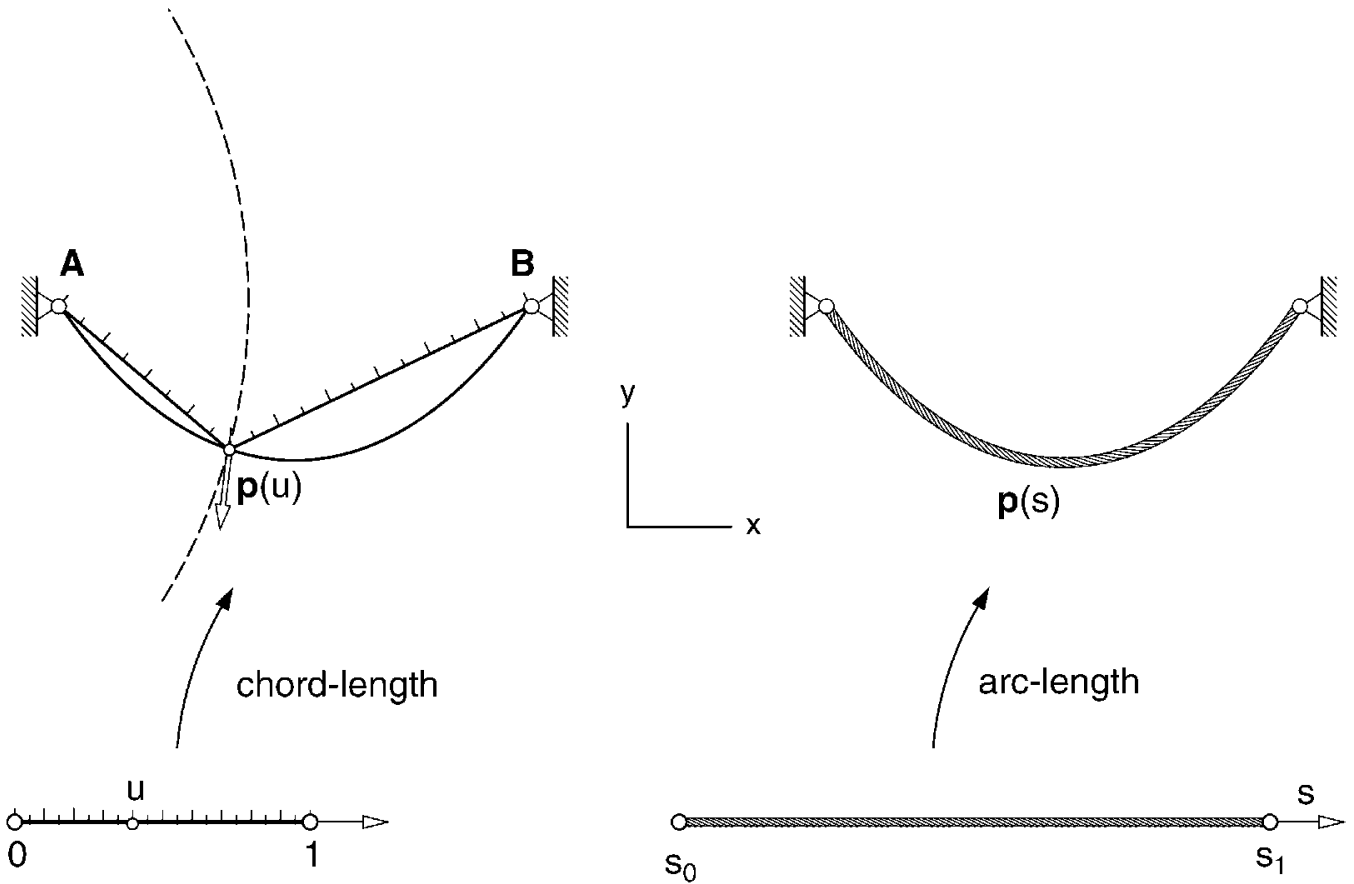

Fig. 1. Chord-length versus arc-length parametrization

in $u$. Compare this rubber-band construction with that of arc-length parametrization, where the parameter domain is represented by an inextensible rope, which is then bent to draw the curve.

Chord-length parametrization has recently received attention in CAGD. Resorting to traditional trigonometry (Sabin and Dodgson, 2005) or symbolic manipulation (Farin, 2006), it has been recently proved that quadratic circles in standard rational Bézier form are parameterized precisely by chord-length. By using bipolar coordinates and complex analysis, we reinterpret chord-length parametrization and check this property in a more natural way.

The article is arranged as follows. First, in Section 2, chord-length parametrization is analyzed within its natural framework, namely bipolar coordinates. In Section 3 we show that the chord-length property of standard Bézier circles admits a very simple proof, since such circles are isoparametric curves in bipolar coordinates. We also determine what Bézier representations of a circle share this property. In Sections 4, 5 we investigate on the existence of other planar and 3D curves enjoying rational chord-length parametrization. Finally, conclusions are drawn in Section 6.

\section{Chord-length parametrization and bipolar coordinates}

\subsection{Interpreting chord-length parametrization}

The chord (1) could be interpreted as a geometric coordinate rather than a property related to a particular curve. Consider two fixed points $\mathbf{A}, \mathbf{B}$, and define the coordinate $u$ of a generic point $\mathbf{p}$ with respect to $\mathbf{A}, \mathbf{B}$ as:

$$
u:=\frac{d_{A}}{d_{A}+d_{B}}, \quad u \in[0,1], d_{A}=|\mathbf{p}-\mathbf{A}|, d_{B}=|\mathbf{p}-\mathbf{B}| .
$$

Given a curve $\mathbf{p}(u)$ over a unit domain $u \in[0,1]$, with $\mathbf{A}=\mathbf{p}(0), \mathbf{B}=\mathbf{p}(1)$, the curve is chord-length parameterized if its parameter $u$ coincides with the coordinate (2). Therefore, such curves admit a trivial inversion (Sederberg and Zheng, 2002): given a point $\mathbf{p}$ on the curve, the corresponding value $u$ is given by the explicit expression (2).

For a fixed value $u$, the equality (2) defines the locus of points $\mathbf{p}$ whose distances from two fixed points $\mathbf{A}, \mathbf{B}$ are in a constant ratio:

$$
\frac{u}{1-u}=\frac{d_{A}}{d_{B}}
$$



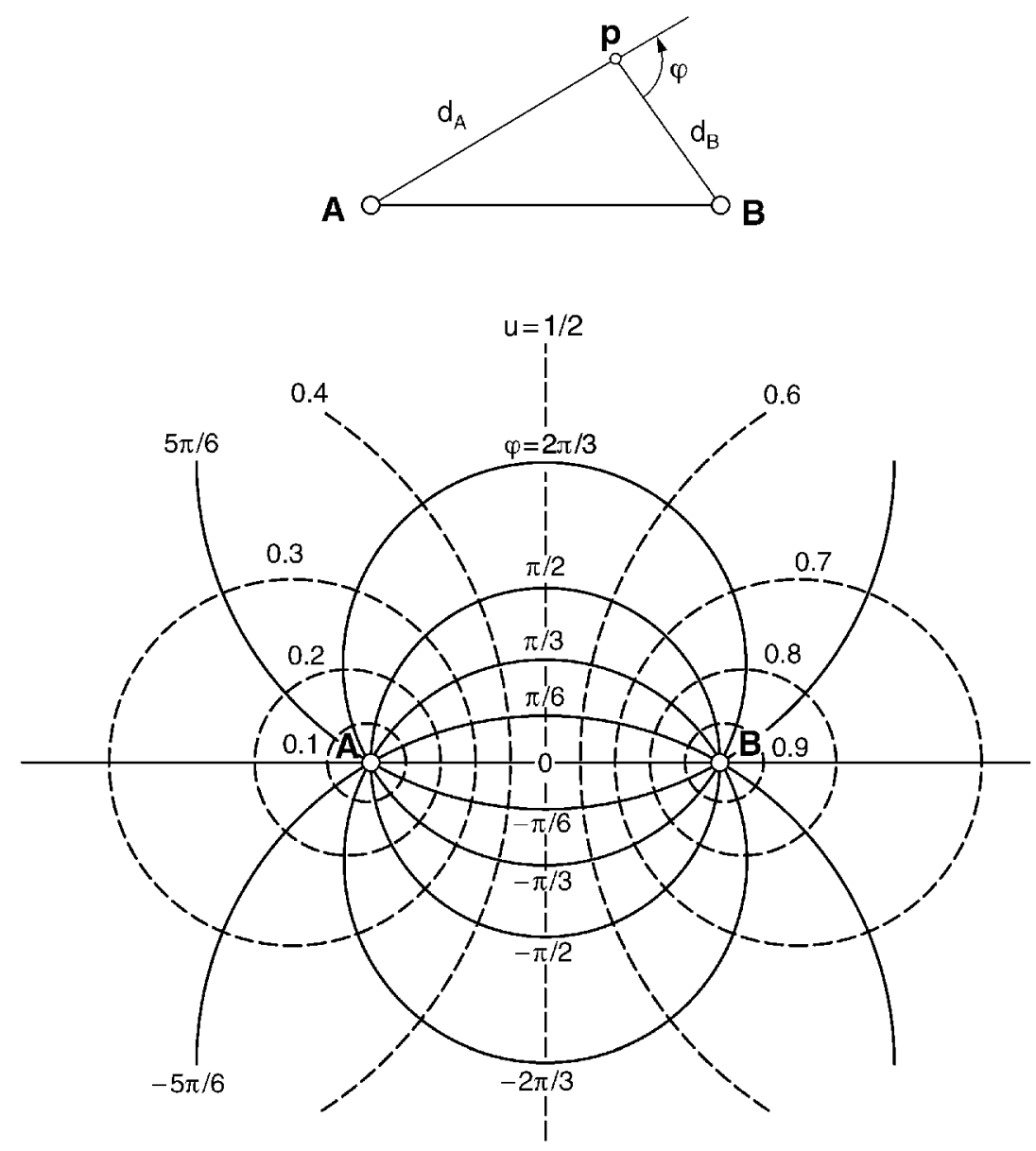

Fig. 2. Bipolar coordinates $(u, \varphi)$ and their isoparametric curves.

Such a locus is a circle (Ogilvy, 1990), a remarkable result due to the Greek geometer Apollonius of Perga. This circle of Apollonius has a centre lying on the line $\mathbf{A B}$ (Fig. 2). For $u=1 / 2$ the circle degenerates to a straight line, the perpendicular bisector of the segment AB.

The use of complex numbers leads to the interpretation of the chord-length property in terms of complex ratios. Given 3 complex points $\mathbf{A}, \mathbf{B}, \mathbf{z}$, we define their ratio as:

$$
[\mathbf{A}, \mathbf{B}, \mathbf{z}]:=\frac{\mathbf{z}-\mathbf{A}}{\mathbf{B}-\mathbf{z}}
$$

which is another complex number, unless the three points are aligned. In consequence, by equality (3), chord-length parametrizations are simply those preserving the (modulus) of the ratios:

$$
[0,1, u]=|[\mathbf{A}, \mathbf{B}, \mathbf{p}(u)]| \text {. }
$$

\subsection{Defining bipolar coordinates}

We are now ready to define the bipolar coordinates $(u, \varphi)$. The second coordinate $\varphi \in(-\pi, \pi]$ of a point $\mathbf{p}$ is the angle between the segments $\mathbf{p B}$ and $\mathbf{A p}$ (Fig. 2). Isoparametric curves with constant $\varphi$ are again circular arcs, the locus of points that see a segment $\mathbf{A B}$ with constant angle $(\pi-\varphi)$. These circular $\operatorname{arcs} \varphi=$ constant form at $\mathbf{A}$ an angle $\varphi$ with the segment $\mathbf{A B}$, and cut orthogonally the iso- $u$ Apollonian circles.

Some warnings regarding terminology. Traditionally, bipolar coordinates are defined using the logarithm $\ln \left(d_{A} / d_{B}\right)$ as coordinate instead of $u(2)$, and the angle $\pi-\varphi$, instead of $\varphi$ (Spiegel and Liu, 1999). However, our customized 
definition proves more convenient to analyze chord-length parametrization. These bipolar coordinates must not be confused with the sometimes called two-centre bipolar coordinates $\left(d_{A}, d_{B}\right)$, which have been employed in a CAGD context by Farouki and Moon (2000).

The transformation from bipolar to Cartesian coordinates is easily carried out using complex notation. Simply consider the geometry of Fig. 2:

$$
\frac{\mathbf{p}-\mathbf{A}}{\mathbf{B}-\mathbf{p}}=\frac{d_{A}}{d_{B}} \mathrm{e}^{\mathrm{i} \varphi},
$$

introduce the relationship (3) and isolate $\mathbf{p}$ in terms of $(u, \varphi)$ :

$$
\mathbf{p}(u, \varphi)=\frac{(1-u) \mathbf{A}+u \mathbf{w} \mathbf{B}}{(1-u)+u \mathbf{w}}, \quad \mathbf{w}=\mathrm{e}^{\mathbf{i} \varphi} .
$$

To construct chord-length parametrized curves $\mathbf{p}(u)$, simply choose an arbitrary function $\varphi(u)$. Such curves can be thus regarded as the analogue, in bipolar coordinates $(u, \varphi)$, of nonparametric curves $(u, f(u))$ in Cartesian coordinates $(x, y)$, where one coordinate is explicitly expressed as a function of the other one.

\subsection{Complex inversion}

Complex inversion deserves our attention, since it provides us with a powerful tool to derive new curves from existing ones. Inversion of centre at the midpoint $\mathbf{O}$ of the segment $\mathbf{A B}$ turns out to be a straightforward operation in bipolar coordinates, if we choose $\mathbf{A}=-1, \mathbf{B}=1$, so that $\mathbf{O}$ lies at the origin $\mathbf{z}=0$. Flipping over the quotient (5), we get a similar expression where $\mathbf{w}$ is replaced by $-\mathbf{w}$. In compact form:

$$
\frac{1}{\mathbf{p}(u, \varphi)}=\mathbf{p}(u, \varphi-\pi), \quad \mathbf{A}=-1, \mathbf{B}=1 .
$$

In consequence, the inverse $1 / \mathbf{p}(u)$ of a chord-length parametrized curve $\mathbf{p}(u)$ has also chord-length parametrization. Formally speaking, the set of chord-length parametrized curves is closed with respect to inversions of centre $\mathbf{O}$. This property certainly applies to iso- $\varphi$ lines: the arc for a constant $\varphi$ transforms via inversion to the complementary one $(\varphi-\pi)$. In other words, the automorphism (6) maps the complete circle to itself.

\section{Circles and chord-length parametrization}

\subsection{Quadratic circles}

Setting a constant $\varphi$, the quotient (5) furnishes a chord-length parametrization $\mathbf{p}(u), u \in[0,1]$, for the corresponding isoparametric curves, namely circular arcs with endpoints $\mathbf{A}=\mathbf{p}(0), \mathbf{B}=\mathbf{p}(1)$. What kind of a parametrization is it? Nothing else than a Möbius transformation, i.e., a complex rational linear map (Needham, 1997), which consequently maps the real line to a circle. After multiplying numerator and denominator by the conjugate denominator, we get indeed a rational quadratic parametrization (with real denominator). Such a parametrization must be the standard one (Bézier endpoints $\mathbf{p}_{0}, \mathbf{p}_{2}$ with unit weights), since the shoulder $\mathbf{S}=\mathbf{p}(1 / 2)$ is precisely the arc midpoint (Fig. 3). We have thus easily checked that standard Bézier arcs are chord-length parameterized.

Note the particular case $\varphi=0$ : the denominator in (5) becomes the unit function, and the circle degenerates to a straight line segment connecting $\mathbf{A}, \mathbf{B}$. This segment is endowed with a linear parametrization, which clearly enjoys the arc-length property as well.

All quadratic $\operatorname{arcs} \mathbf{c}(v)$ stem from the standard $\operatorname{arc} \mathbf{p}(u)$ by rational linear reparametrization $u(v)$. As a result, the shoulder point $\mathbf{c}(1 / 2)$ no longer lies on the midpoint of the arc, and chord-length parametrization is destroyed. For completeness, we give the explicit expression for such non-standard arcs. Consider again that a Möbius map transforms the real line to a circle, and find the specific map $\mathbf{c}(v)$ that transforms $\{0,1,1 / 2\}$ to three distinct complex points $\{\mathbf{a}, \mathbf{b}, \mathbf{s}\}$. Simply write a rational linear function in Bézier form, of endpoints $\mathbf{a}, \mathbf{s}$, and find the weight that results from the condition $\mathbf{s}=\mathbf{c}(1 / 2)$ :

$$
\mathbf{c}(v)=\frac{(1-v) \mathbf{a}+v \omega \mathbf{b}}{(1-v)+v \omega}, \quad \boldsymbol{\omega}=\frac{\mathbf{s}-\mathbf{a}}{\mathbf{b}-\mathbf{s}} .
$$




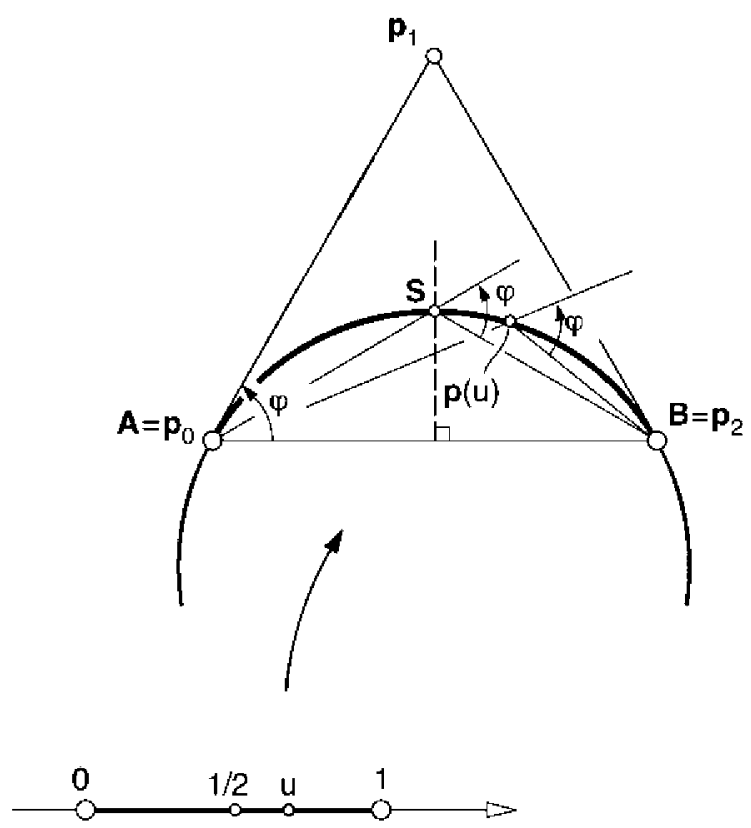

Fig. 3. Standard parametrization of a circle via Möbius map.

\subsection{Rational representations of higher degree}

Regarding Bézier representations of higher degree, Berry proved that any Bézier circle other than quadratic is degenerate (Berry and Patterson, 1997; Sánchez-Reyes, 1997). There exist two types (Sederberg, 1984) of degenerate circles:

1- Improperly parameterized (Sederberg, 1986), generated from the standard quadratic circle via a nonlinear rational parameter substitution. This operation alters the parametrization, which no longer satisfies the chord-length condition.

2- Those with base points, introduced by multiplying the quadratic circle in projective space by an arbitrary polynomial, so that all homogeneous polynomials contain a common factor that can be divided out. Such an operation is called generalized degree elevation (Denker and Herron, 1997), since standard degree elevation corresponds to the limit case of a base point at infinity (Farin, 1999). This procedure does not alter the parametrization, thereby preserving chord-length.

We conclude that, as Farin (2006) noted, the standard quadratic parametrization is the only rational chord-length parametrization of the circle. However, there exist other chord-length Bézier representations, namely those of higher degree derived from the standard one by inserting base points. For the cubic and quartic cases, such representations are subsumed in the formulae given by Chou (1995).

\section{Planar rational curves with chord-length parametrization}

\subsection{Quartics and their shape handles}

We explore now what other planar curves $\mathbf{p}(u)$ admit rational chord-length parametrization on $u \in[0,1]$. Such curves must conform to expression (5), where $\mathbf{w}(u)$ denotes a rational curve on a unit circle:

$$
\mathbf{p}(u)=\frac{(1-u) \mathbf{A}+u \mathbf{w}(u) \mathbf{B}}{(1-u)+u \mathbf{w}(u)}, \quad|\mathbf{w}(u)|=1 .
$$




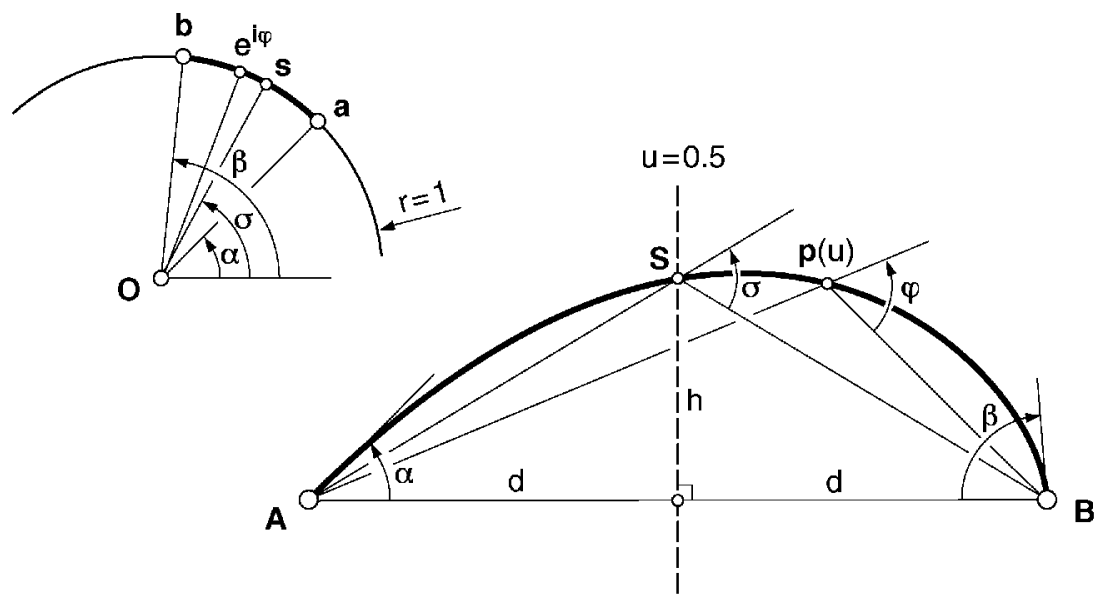

Fig. 4. Angles $\alpha, \beta, \sigma$ defining a chord-length quartic.

To get complex rational quadratic functions (i. e., quartics), just employ a linear map (7) generating a unit circle $\mathbf{w}(u)$. To guarantee a unit radius, choose endpoints $\mathbf{a}=\mathrm{e}^{\mathbf{i} \alpha}, \mathbf{b}=\mathrm{e}^{\mathbf{i} \beta}$ and shoulder point $\mathbf{s}=\mathrm{e}^{\mathbf{i} \sigma}$ of unit modulus. Note that $\mathbf{a}, \mathbf{b}, \mathbf{s}$ must be distinct to avoid divisions by zero in the quotients (7), which implies distinct angles $\alpha, \beta, \sigma$. In the limiting case of both endpoints, the angle $\varphi(u)$, such that $\mathbf{w}(u)=\mathrm{e}^{\mathbf{i} \varphi(u)}$, becomes the angle between the tangent to the curve and the segment $\mathbf{A B}$. We thus control the quartic using the following shape handles (Fig. 4):

a) Endpoints $\mathbf{A}, \mathbf{B}$, and angles $\alpha, \beta$ between the endpoint tangents and the segment $\mathbf{A B}$.

b) Angle $\sigma$ between chords $\mathbf{A S}$ and $\mathbf{S B}$ at $\mathbf{S}=\mathbf{p}(1 / 2)$. Equivalently, we may choose the position of $\mathbf{S}$ on the bisector of $\mathbf{A B}$, by setting the height:

$$
h=d \tan \frac{\sigma}{2}, \quad d=\frac{1}{2}|\mathbf{B}-\mathbf{A}| .
$$

In fact, Fig. 4 is nothing else than the generalization of Fig. 3 for the case of a varying angle $\varphi(u)$. Finally, note that, to construct the inverse curve $1 / \mathbf{p}(u)(6)$, simply choose angles $\alpha-\pi, \beta-\pi, \sigma-\pi$.

\subsection{Equilateral hyperbola and Lemniscate of Bernoulli}

The denominator in the quotient (8) vanishes only for the singular case $\sigma=\pi$, which implies $h=\infty$ (9), yielding an asymptotic behaviour at $\mathbf{S}$. If $\beta=-\alpha$, the quotient takes a simple form, where the denominator becomes a real function:

$$
\mathbf{p}(u)=\frac{\mathbf{A}(1-u)^{2}+\mathbf{a}(\mathbf{B}-\mathbf{A})(1-u) u-\mathbf{B} u^{2}}{(1-u)^{2}-u^{2}} .
$$

This is indeed a quadratic rational curve, i. e., a conic section. A closer inspection of its behaviour, with asymptotic directions of angles $(\alpha+\pi) / 2(u \rightarrow 1 / 2)$ and $\alpha / 2(u \rightarrow \infty)$ with respect to the line $\mathbf{A B}$, reveals that the conic is a (rotated) equilateral hyperbola centred at the midpoint $\mathbf{O}$ of the segment $\mathbf{A B}$ (Fig. 5). We have thus obtained a simple, yet weird Bézier form of the hyperbola, with weights $\{1,0,-1\}$, endpoints $\mathbf{A}, \mathbf{B}$ and central Bézier point at infinity (Farin, 2001), the vector $\mathbf{a}(\mathbf{B}-\mathbf{A}) / 2$. Surprisingly, this parametrization, between two points $\mathbf{A}, \mathbf{B}$ on different branches with parallel tangents, is chord-length.

More appealing is the inverse curve (6). Swapping numerator and denominator (10) results in the $\infty$-shaped figure displayed in Fig. 5, a remarkable quartic known as Lemniscate of Bernoulli (Lawrence, 1972).

\subsection{Limaçon of Pascal}

A special family of curves is generated by setting $\alpha=-\beta=\pi / 2$, and varying $\sigma$ (Fig. 6). The segment $u \in[0,1]$ corresponds to one half of the complete quartic, which is symmetric with respect to the axis $\mathbf{A B}$, and has a double 

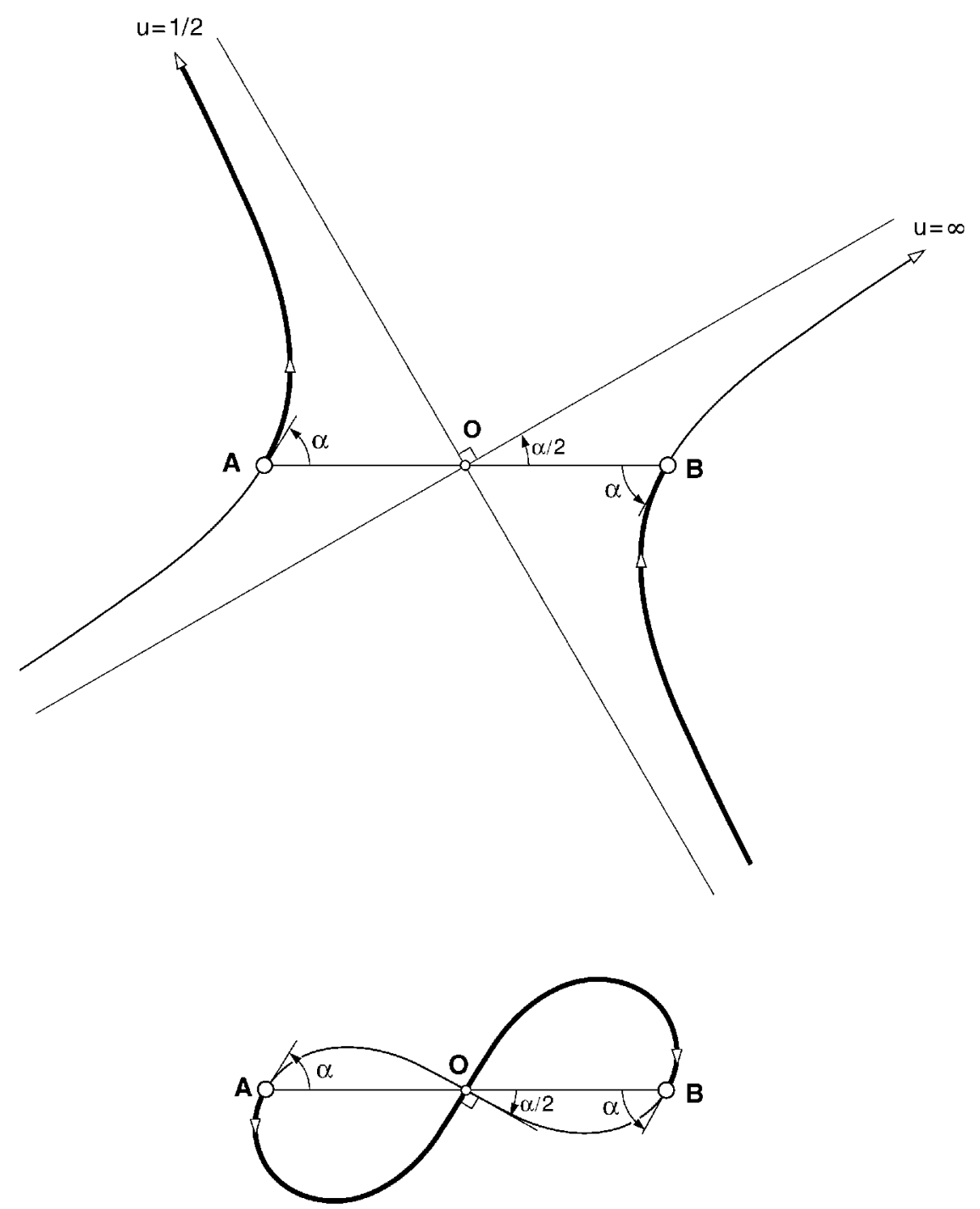

Fig. 5. Equilateral hyperbola and its inverse, the Lemniscate of Bernoulli.

point $\mathbf{D}$ on this line. For $\sigma=0$, we have the Lemniscate of Bernoulli, and as $\sigma$ increases, $\mathbf{D}$ moves along the axis. When $\sigma=\pi / 2$, $\mathbf{D}$ reaches $\mathbf{B}$ and we have a circle, if we set $\mathbf{w}(u)=\mathrm{e}^{\mathbf{i} \pi / 2}=\mathbf{i}$ when computing the quartic (8). For $\sigma>\pi / 2$, $\mathbf{D}$ moves further on to the right, eventually to infinity $(\sigma=\pi)$, which yields an equilateral hyperbola.

The particular case $\sigma=\arccos (-1 / 3)$ is worth studying (Fig. 7). Choosing $\mathbf{A}=-1, \mathbf{B}=1$ for simplicity, after some algebraic manipulation the quartic decomposes as:

$$
\mathbf{p}(u)=L+\mathbf{c}(u)-L \mathbf{c}^{2}(u), \quad L=\frac{\sqrt{2}}{2},
$$

where $\mathbf{c}(u)$ denotes a Möbius map (7) defining a non-standard circle, of endpoints $\mathbf{a}=-1, \mathbf{b}=1$ and shoulder $\mathbf{s}=\mathrm{e}^{\mathrm{i} 3 \pi / 4}$. A linear combination (11) of a circle and its power generates an epitrochoid (Lawrence, 1972), the path traced by a point $\mathbf{p}$ attached to a moving circle as it roles without sliding on the outside of a fixed circle. In this case, the point on the moving circle is at distance $L$ from its centre, and the fixed circle is centred at $L$. In addition, both circles have an equal (unitary) diameter, thereby yielding an instance of a special curve called Limaçon of Pascal.

A rational representation of epitrochoids was previously studied by Sánchez-Reyes (1999). However, it was achieved combining standard Bézier circles and, in consequence, the chord-length parametrization (11) for the Limaçon, involving a non-standard circle, was not encompassed. 


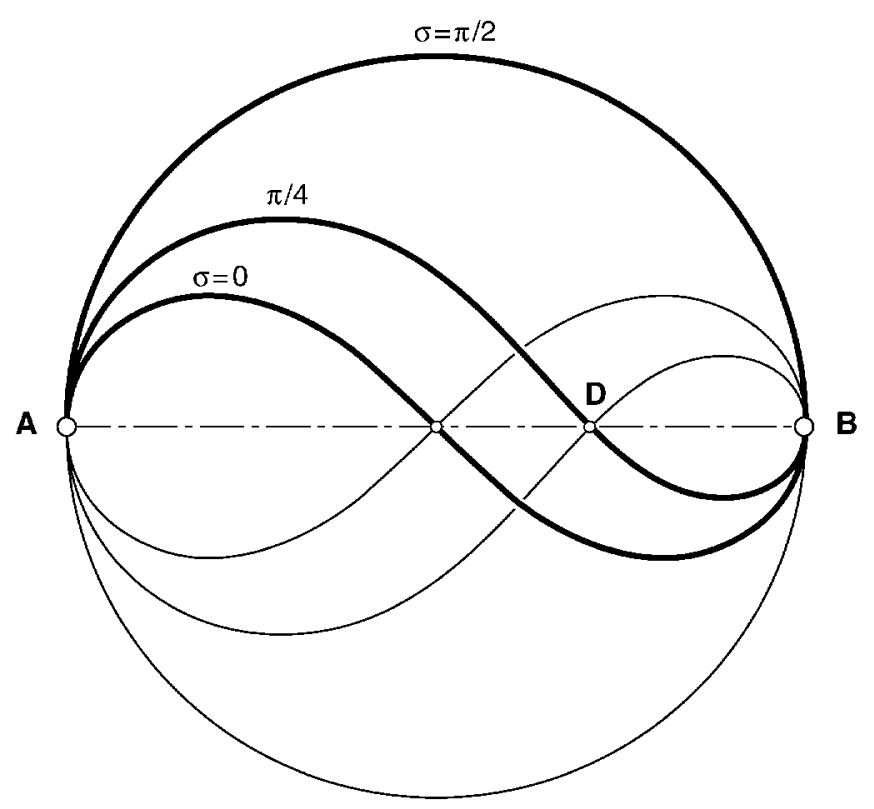

Fig. 6. One-parameter family of quartics, of constant $\alpha=-\beta=\pi / 2$ and a varying $\sigma \in[0, \pi / 2 \mid$.

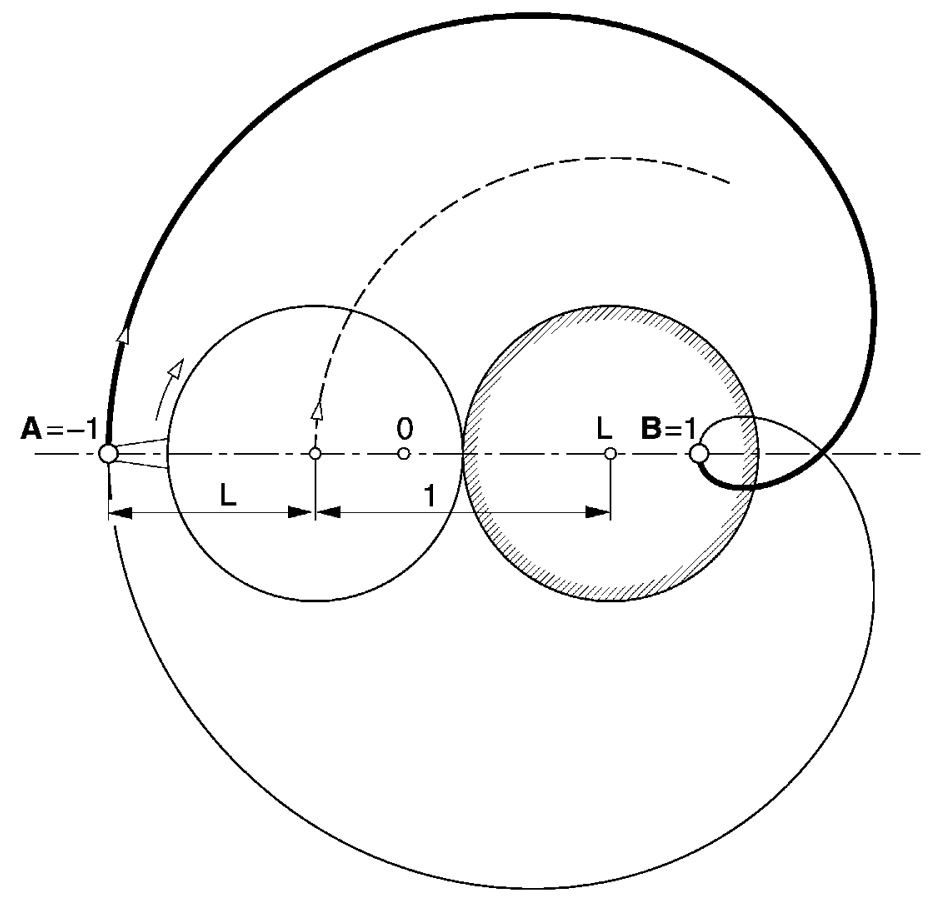

Fig. 7. Limaçon of Pascal as an epitrochoid: $\alpha=-\beta=\pi / 2, \sigma=\operatorname{arcos}(-1 / 3)$.

\section{Space curves with chord-length parametrization}

Bipolar coordinates $(u, \varphi)$ naturally extend to 3D by adding a third coordinate $\theta$, corresponding to a rotation around the axis $\mathbf{A B}$. Apollonian circles become spheres $u=$ constant, and circles of constant $\varphi$ become toroidal isosurfaces. 
We address now the case of 3D curves $\mathbf{q}(u)$ with arc-length parametrization. To fix notation, suppose that $\mathbf{A B}$ defines the $x$-axis. Clearly, the rotation $\theta$ is immaterial for chord-length, and a 3D curve of Cartesian coordinates $\mathbf{q}(u)=\{x(u), y(u), z(u)\}$ is chord-length only if this property is shared by its associated planar curve $\mathbf{p}(u)$ :

$$
\mathbf{p}(u)=x(u)+\mathbf{i} r(u), \quad r(u)=\sqrt{y^{2}(u)+z^{2}(u)} .
$$

Equivalently, we construct a 3D curve $\mathbf{q}(u)$ from any 2D chord-length parametrized curve $\mathbf{p}(u)(12)$, by choosing any pair of functions $y(u), z(u)$ such that $r, y, z$ are Pythagorean triples.

Regarding rational curves, we could easily generate a $3 \mathrm{D}$ rational curve $\mathbf{q}(u)$ via product of $\mathbf{p}(u)$ with a rational unit circle $\mathbf{c}(u)$ :

$$
\mathbf{q}(u)=\{x(u), r(u) Y(u), r(u) Z(u)\}, \quad \mathbf{c}(u)=\{Y(u), Z(u)\} .
$$

Geometrically, we rotate each point $\mathbf{p}(u)$ around the $x$-axis the angle indicated by $\mathbf{c}(u)$ and obtain $\mathbf{q}(u)$. For the simple case of both $\mathbf{p}(u), \mathbf{c}(u)$ quadratic circles, we get a quartic $\mathbf{q}(u)$ that lies on a toroidal isosurface of constant $\varphi$. The special instances $\varphi= \pm \pi / 2$ of a semicircle $\mathbf{p}(u)$ yield a curve on a sphere connecting two poles $\mathbf{A}, \mathbf{B}$.

\section{Conclusions}

We have elucidated the intimate connection between bipolar coordinates and curves with chord-length parametrization. Such curves are simply those whose parameter coincides with one of the coordinates, so that chord-length parametrized curves can be regarded as the analogue of nonparametric curves. Remarkably, they have a trivial inversion algorithm since the parameter corresponding to a given point is given by a simple explicit expression.

Whereas the straight line is the only curve admitting a parametrization of rational functions of its arc length (Farouki and Sakkalis, 1991, 2007), the variety of rational chord-length parametrized curves is much higher. In addition to the straight line, circle and equilateral hyperbola, there exist planar and space quartics displaying chord-length parametrization, which include the Lemniscate of Bernoulli and an instance of the Limaçon of Pascal. Such planar quartics admit a compact complex parametrization (rational quadratic, with complex denominator), intrinsically simpler than the traditional one (quartic with real denominator).

\section{Acknowledgements}

This work is supported by the Spanish Ministerio de Educación y Ciencia, under research grant DPI2006-09091.

\section{References}

Berry, T.G., Patterson, R.R., 1997. The uniqueness of Bézier control points. Computer Aided Geometric Design 14 (4), $877-879$.

Chou, J.J., 1995. Higher order Bézier circles. Computer-Aided Design 27 (4), 303-309.

Denker, W.A., Herron, G.J., 1997. Generalizing rational degree elevation. Computer Aided Geometric Design 14 (5), $399-406$.

Farin, G., 1999. NURB Curves and Surfaces: from Projective Geometry to Practical Use, second ed. AK Peters.

Farin, G., 2001. Curves and Surfaces for Computer Aided Geometric Design, fifth ed. Morgan Kaufmann.

Farin, G., 2006. Rational quadratic circles are parametrized by chord length. Computer Aided Geometric Design 23 (9), $722-724$.

Farouki, R.T., Moon, H.P., 2000. Bipolar and multipolar coordinates. In: Proceedings of the 9th IMA Conference on the Mathematics of Surfaces, pp. 348-371.

Farouki, R.T., Sakkalis, T.J., 1991. Real rational curves are not unit speed. Computer Aided Geometric Design 24 (2), $151-157$.

Farouki, R.T., Sakkalis, T.J., 2007. Rational space curves are not "unit speed". Computer Aided Geometric Design 24 (4), $238-240$.

Lawrence, J.D., 1972. A Catalog of Special Plane Curves. Dover, New York.

Needham, T., 1997. Visual Complex Analysis. Clarendon Press, Oxford.

Ogilvy, C.S., 1990. Excursions in Geometry. Dover.

Sabin, M., Dodgson, N., 2005. A circle-preserving variant of the four-point subdivision scheme. In: Lyche, T., Schumaker, L. (Eds.), Mathematical Methods for Curves and Surfaces. Troms $\varnothing 2004$. Nashboro Press, pp. 275-286.

Sánchez-Reyes, J., 1997. Higher-order Bézier circles. Computer-Aided Design 29 (6), 469-472.

Sánchez-Reyes, J., 1999. Bézier representation of epitrochoids and hypotrochoids. Computer-Aided Design 31, 747-750.

Sederberg, T.W., 1984. Degenerate parametric curves. Computer Aided Geometric Design 1 (4), 301-307.

Sederberg, T.W., 1986. Improperly parametrized rational curves. Computer Aided Geometric Design 3 (1), 67-75.

Sederberg, T.W., Zheng, J., 2002. Algebraic Methods for Computer Aided Geometric Design. In: Farin, G., Hoschek, J., Kim, M.-S. (Eds.), Handbook of Computer Aided Geometric Design. North Holland/Elsevier, pp. 363-387.

Spiegel, M.R., Liu, J., 1999. Mathematical Handbook of Formulas and Tables, second ed. McGraw-Hill. 